\title{
Worst-Case Error Probability of a Spread-Spectrum System in Energy-Limited Interference
}

\author{
Murad Hizlan \\ Cleveland State University, hizlan@cxvax.csuohio.edu \\ Brian L. Hughes \\ North Carolina State University at Raleigh, blhughes@eos.ncsu.edu
}

Follow this and additional works at: https://engagedscholarship.csuohio.edu/enece_facpub

Part of the Systems and Communications Commons

How does access to this work benefit you? Let us know!

\section{Publisher's Statement}

(C) 1998 IEEE. Personal use of this material is permitted. Permission from IEEE must be obtained for all other uses, in any current or future media, including reprinting/republishing this material for advertising or promotional purposes, creating new collective works, for resale or redistribution to servers or lists, or reuse of any copyrighted component of this work in other works.

\section{Original Citation}

Hizlan, M., \& Hughes, B. L. (February 01, 1998). Worst-case error probability of a spread-spectrum system in energy-limited interference. IEEE Transactions on Communications, 46, 2, 286-296.

\section{Repository Citation}

Hizlan, Murad and Hughes, Brian L., "Worst-Case Error Probability of a Spread-Spectrum System in Energy-Limited Interference" (1998). Electrical Engineering \& Computer Science Faculty Publications. 80.

https://engagedscholarship.csuohio.edu/enece_facpub/80

This Article is brought to you for free and open access by the Electrical Engineering \& Computer Science Department at EngagedScholarship@CSU. It has been accepted for inclusion in Electrical Engineering \& Computer Science Faculty Publications by an authorized administrator of EngagedScholarship@CSU. For more information, please contact library.es@csuohio.edu. 


\title{
Worst-Case Error Probability of a Spread-Spectrum System in Energy-Limited Interference
}

\author{
Murad Hizlan, Member, IEEE, and Brian L. Hughes, Member, IEEE
}

\begin{abstract}
We consider a communication channel corrupted by thermal noise and by an unknown and arbitrary interference of bounded energy. For this channel, we derive a simple upper bound to the worst-case error probability suffered by a directsequence (DS) communication system with error-correction coding, pseudorandom interleaving, and a correlation receiver. This bound is exponentially tight as the block length of the errorcorrecting code becomes large. Numerical examples are given that illustrate the dependence of the bound on the choice of errorcorrecting code, the type of interleaving used, and the relative energy of the Gaussian noise and arbitrary interference.
\end{abstract}

Index Terms - Direct-sequence modulation, robust detection, signal detection, spread-spectrum.

\section{INTRODUCTION}

$\mathbf{O}$ NE OF THE most important properties of spreadspectrum communication systems is the ability to suppress a wide variety of interfering signals. For directsequence (DS) modulation, this ability has been investigated for several "canonical" forms of interference, including stationary Gaussian noise [11], pulsed Gaussian noise [15], tones [5], [13], narrowband noise [9], [12], [16], impulsive noise [1], [2], and multiple-access interference, e.g., [5], [14].

In certain communication situations (e.g., hostile jamming), it is of interest to determine the worst possible error probability that can be inflicted on a DS system by an interfering signal with a given energy, and to identify the type of interference that achieves it. It is far from obvious, however, that this worstcase interference is among the canonical forms of interference mentioned above.

In [7], we derived an upper bound for the worst-case error probability of a DS modulator and a linear correlation receiver when used on a channel corrupted by Gaussian noise and an arbitrary interfering signal of bounded energy. The worst-case interference for this communication system was shown to be

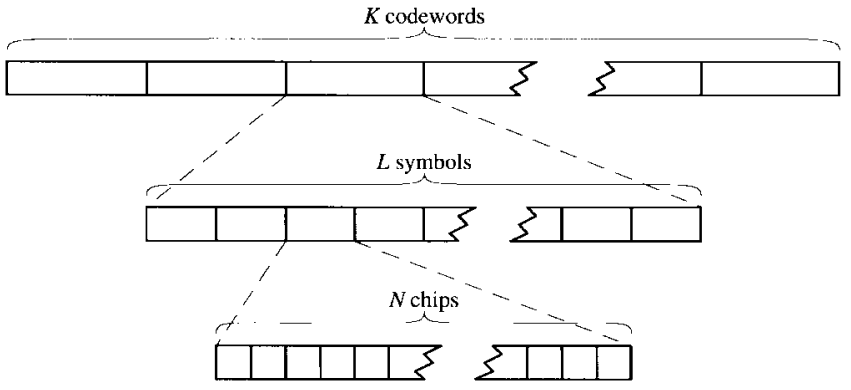

Fig. 1. Symbolic representation of the DS signal.

a tone with the same frequency and phase as the DS carrier. This improved upon an earlier bound by Kullstam [10].

The aim of this paper is to generalize the results in [7] to a DS system with error-control coding, pseudorandom interleaving, and a correlation receiver. In Section II, we describe the channel and communication system models precisely, and we give an expression for the worst-case error probability. In Section III, we derive a simple upper bound to this error probability for a channel corrupted by additive white Gaussian noise (AWGN) and an unknown arbitrary interference of bounded energy. This bound is exponentially tight as the block length of the code becomes large. In Section IV, we identify the type of interference that maximizes the bound and give numerical examples that illustrate the dependence of the bound on the choice of code parameters, the type of interleaving, and the relative energy of the Gaussian noise and arbitrary interference. Finally, our conclusions are summarized in Section V.

\section{System Model AND Definitions}

We consider a communication situation in which a DS signal with error-correction coding and pseudorandom block interleaving is transmitted over a communication channel corrupted by thermal noise and an arbitrary energy-limited interference. In this section, we first introduce a waveform model for this communication system. We then derive a simpler, but equivalent, matrix channel model. Finally, we present a measure of the worst-case system performance.

\section{A. A Waveform Model}

A symbolic representation of the transmitted signal is given in Fig. 1. Every $K T$ seconds, the transmitter generates $K$ messages $m_{i}, i=1, \cdots, K$, taking values in $\{1, \cdots, M\}$. Each message is encoded by a binary block code with block length $L$ and transmitted by DS modulation with $N$ pseudonoise 
chips per code symbol. Prior to transmission, the $K L$ code symbols are permuted by pseudorandom block interleaving. By symmetry, each of the $K$ messages will suffer the same error probability. Thus, without loss of generality, we may concentrate on only one of the $K$ messages, say $m_{1}$, and call it $m$.

Denote the binary $\{ \pm 1\}$ codeword associated with message $m$ by $\mathbf{x}^{(m)} \triangleq\left[x_{0}^{(m)}, \cdots, x_{L-1}^{(m)}\right]$. The symbols of the codeword $\mathbf{X}^{(m)}$ are pseudorandomly interleaved over $K$ codewords to form an interleaved code waveform

$$
Z^{(m)}(t) \triangleq \sum_{\ell=0}^{L-1} x_{\ell}^{(m)} u\left(t-J_{\ell} T_{S}\right), \quad 0 \leq t<K T
$$

where $u(t)=1$ in the interval $\left[0, T_{S}\right)$ and vanishes outside, and $T_{S} \triangleq T / L$ is the symbol duration. The index sequence $\left\{J_{0}, \cdots, J_{L-1}\right\}$ in (1) represents pseudorandom block interleaving of the symbols of $\mathrm{x}^{(m)}$ over $K$ codewords, and is modeled as a random sequence which is uniformly distributed over all permutations of size $L$ of the sequence $\{0,1, \cdots, L K-1\}$.

The transmitted signal $X^{(m)}(t)$ is obtained from $Z^{(m)}(t)$ by binary phase-shift keying (BPSK) modulation and DS spreading

$$
\begin{aligned}
& X^{(m)}(t) \triangleq \sqrt{2 E / T} \cos (\omega t) C(t) Z^{(m)}(t), \\
& 0 \leq t<K T
\end{aligned}
$$

where $E$ is the energy per codeword at the receiver, $T_{C} \triangleq T_{S} / N$ is the chip duration, $\omega$ is the carrier frequency with $\omega \gg 2 \pi T_{C}^{-1}$, and $C(t)$ is the spreading waveform

$$
C(t) \triangleq \sum_{i=0}^{N L K-1} A_{i} v\left(t-i T_{C}\right), \quad 0 \leq t<K T .
$$

Here, $v(t)$ is a low-pass chip waveform that satisfies $\int_{0}^{T_{C}} v^{2}(t) d t=T_{C}$ and vanishes outside the interval $\left[0, T_{C}\right)$.

The pseudonoise sequence $\left\{A_{i}\right\}$ is modeled as an independent identically distributed (i.i.d.) sequence of random variables which satisfy $\operatorname{Pr}\left\{A_{i}=+1\right\}=\operatorname{Pr}\left\{A_{i}=-1\right\}=1 / 2$ and are independent of $\left\{J_{0}, \cdots, J_{L-1}\right\}$. We remark here that, although (2) models transmission of the encoded symbols by binary phase-shift keying, all of the results in this paper can be adapted in a straightforward manner to quadriphase-shift keying (QPSK).

As illustrated in Fig. 2, the received signal takes the form

$$
Y(t) \triangleq X^{(m)}(t)+W(t)+S(t), \quad 0 \leq t<K T
$$

where $W(t)$ is a zero-mean white Gaussian noise process with one-sided power spectral density $N_{0} \mathrm{~W} / \mathrm{Hz}$, and $S(t)$ is an unknown and arbitrary interfering signal. The signal $S(t)$ represents interference from sources with unknown statistics, such as jamming, multiple-access interference, and impulsive noise. In this paper, we consider a communication situation in which nothing is known about $S(t)$ except that it is independent of $\left\{A_{i}\right\}, W(t)$, and $\left\{J_{0}, \cdots, J_{L-1}\right\}$, and that its energy is constrained. Thus, $S(t)$ may be random or

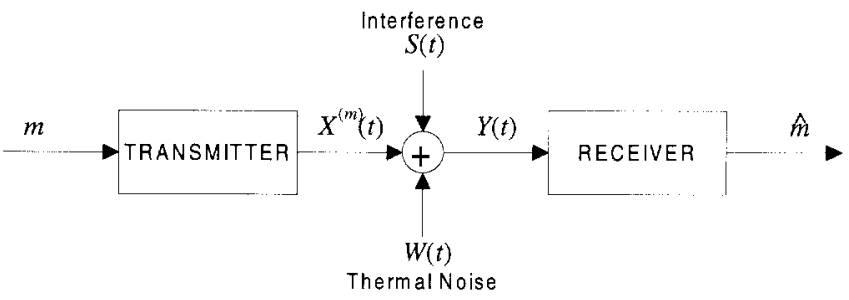

Fig. 2. Waveform channel model.

deterministic, narrow-band or wide-band, stationary or timevarying, Gaussian or non-Gaussian.

To bound the error probability of a receiver for (3), we must place some constraint on the interference energy $\int_{0}^{K T} S^{2}(t) d t$. This paper will focus on constraints which consist of bounds on the moments or tails of the probability distribution of this energy. Throughout most of the paper, we focus attention on the particular case when interference energy is strictly bounded, i.e.,

$$
\int_{0}^{K T} S^{2}(t) d t \leq K T P_{I}, \quad \text { almost surely (a.s.). }
$$

The relationship of this case to other energy constraints is described in Section II-D.

At the receiver, signal detection is performed by a standard correlation receiver comprising $M$ coherent filters, each one matched to one of the $M$ possible transmitted codewords. The decision is the index of the largest correlation receiver output

$$
\hat{m} \triangleq \arg \max _{1 \leq i \leq M} U_{i}
$$

where ties are resolved randomly and $U_{i} \triangleq \int_{0}^{K T} Y(t) X^{(i)}(t) d t$.

\section{B. An Equivalent Matrix Channel}

It is convenient to recast the waveform channel described above into an equivalent matrix form. Let $\mathbf{S} \triangleq\left(S_{0}, \cdots, S_{N L K-1}\right)$, where $S_{i} \triangleq \int_{0}^{K T} S(t) \varphi_{i}(t) d t$ denotes the projection of $S(t)$ onto the set of orthonormal signals

$$
\begin{aligned}
& \varphi_{i}(t) \triangleq \sqrt{2 / T_{C}} \cos (\omega t) v\left(t-i T_{C}\right), \\
& 0 \leq t<K T, \quad 0 \leq i<N L K .
\end{aligned}
$$

Now observe that the $N L$ waveforms $\varphi_{J_{\ell} N+n}(t), 0 \leq$ $\ell<L, 0 \leq n<N$ form an orthonormal basis for $X^{(1)}(t), \cdots, X^{(M)}(t)$. By projecting $Y(t), X^{(m)}(t), W(t)$, and $S(t)$ in (3) onto this basis, we find that the waveform channel of Fig. 2 is equivalent to the matrix channel

$$
\mathbf{Y} \triangleq \mathbf{V}^{(m)}+\mathbf{W}+\hat{\mathbf{S}} \text {. }
$$

Here, $\quad \mathbf{V}^{(m)}$ is an $L \times N$ matrix with elements $V_{\ell, n}^{(m)} \triangleq \sqrt{E / L N} x_{\ell}^{(m)} A_{J_{\ell} N+n}, 0 \leq \ell<L, 0 \leq n<N$. The thermal noise becomes an $L \times N$ matrix $\mathbf{W}$ with i.i.d. $\mathcal{N}\left(0, N_{0} / 2\right)$ elements, and the unknown interference becomes an $L \times N$ matrix $\hat{\mathbf{S}}$ with elements $\hat{S}_{\ell, n} \triangleq S_{J_{\ell} N+n}$, $0 \leq \ell<L, 0 \leq n<N$. 
With these definitions, the correlation receiver (5) can be rewritten as

$$
\hat{m} \triangleq \arg \max _{1 \leq i \leq M}\left\langle\mathbf{Y}, \mathbf{V}^{(i)}\right\rangle
$$

where $\langle\cdot, \cdot\rangle$ denotes the inner product.

\section{Worst-Case Probability of Error}

Given $\mathbf{S}=\mathbf{s}$, the conditional probability of error of the receiver in (5) and (7) is given by

$$
\varepsilon\left(\mathbf{s}, \nu^{2}\right) \triangleq \frac{1}{M} \sum_{m=1}^{M} \operatorname{Pr}\{\hat{m} \neq m \mid \mathbf{S}=\mathbf{s}\}
$$

where $\nu^{2} \triangleq 2 E / N L N_{0}$ is the chip signal-to-noise power ratio. For a particular $\mathbf{S}$, the (unconditional) probability of error is then $\mathbf{E}\left\{\varepsilon\left(\mathbf{S}, \nu^{2}\right)\right\}$, where $\mathbf{E}$ denotes expectation with respect to the distribution of $\mathbf{S}$.

Our objective in this paper is to develop tools for bounding the error probability when nothing is known about $\mathbf{S}$ except a constraint on the energy of $S(t)$. Note that the energy constraint (4) implies that $\mathbf{S}$ satisfies

$$
P(\mathbf{S}) \triangleq \frac{1}{N L K} \sum_{i=0}^{N L K-1} S_{i}^{2} \leq E / N L \sigma^{2} \text { (a.s.) }
$$

where $\sigma^{2} \triangleq E / T P_{I}$ is the signal-to-interference power ratio. Hence, the worst-case probability of error for all interfering signals satisfying (4) is

$$
\mathcal{E}\left(\sigma^{2}, \nu^{2}\right) \triangleq \sup _{\mathbf{S}: P(\mathbf{S}) \leq E / N L \sigma^{2}} \mathbf{E}\left\{\varepsilon\left(\mathbf{S}, \nu^{2}\right)\right\}
$$

Note that this probability will not be changed by restricting the supremum to deterministic sequences s, i.e.,

$$
\mathcal{E}\left(\sigma^{2}, \nu^{2}\right)=\sup _{\mathbf{s}: P(\mathbf{s}) \leq E / N L \sigma^{2}} \varepsilon\left(\mathbf{s}, \nu^{2}\right) .
$$

To see this, observe that the right side of (10) is clearly a lower bound to $\mathcal{E}\left(\sigma^{2}, \nu^{2}\right)$ since deterministic sequences are a particular case of random sequences. Conversely, it is also an upper bound for $\mathcal{E}\left(\sigma^{2}, \nu^{2}\right)$ because it is greater than or equal to $\varepsilon\left(\mathbf{S}, \nu^{2}\right)$ for every outcome $\mathbf{S}$ that satisfies $P(\mathbf{S}) \leq E / N L \sigma^{2}$.

The main contribution of this paper is to derive an upper bound for $\mathcal{E}\left(\sigma^{2}, \nu^{2}\right)$.

\section{Other Constraints on Interference Energy}

Throughout most of this paper, we consider a communication situation in which interference energy is strictly bounded, as in (4) and (9). This model would be appropriate when the source of interference is a single energy-limited transmitter or jammer. However, many types of interference of practical importance will not satisfy (9) for any choice of $\sigma^{2}$ (e.g., Gaussian noise). In this section, we show how $\mathcal{E}\left(\sigma^{2}, \nu^{2}\right)$ may be used to bound the error probability for a variety of situations where (9) is violated, but where a bound on the tail probability or moments of $P(\mathbf{S})$ is known.
First, let $\mathbf{S}$ be any sequence of interference samples. We can use $\mathcal{E}\left(\sigma^{2}, \nu^{2}\right)$ to bound the conditional error probabilities

$$
\mathbf{E}\left\{\varepsilon\left(\mathbf{S}, \nu^{2}\right) \mid P(\mathbf{S})=p\right\} \leq \mathcal{E}\left(E / N L p, \nu^{2}\right), \quad p \geq 0
$$

regardless of whether $\mathbf{S}$ satisfies (9). If the distribution function of $P(\mathbf{S})$ were known, say $F(p) \triangleq \operatorname{Pr}\{P(\mathbf{S}) \leq p\}$, then

$$
\mathbf{E}\left\{\varepsilon\left(\mathbf{S}, \nu^{2}\right)\right\} \leq \int_{0}^{\infty} \mathcal{E}\left(E / N L p, \nu^{2}\right) d F(p) .
$$

A straightforward consequence of (11) is the following bound on the error:

$$
\mathbf{E}\left\{\varepsilon\left(\mathbf{S}, \nu^{2}\right)\right\} \leq \min _{p \geq 0} \mathcal{E}\left(E / N L p, \nu^{2}\right)+\operatorname{Pr}\{P(\mathbf{S})>p\} .
$$

Thus, in any situation where $F(p)$ or bounds on the tail probabilities $\operatorname{Pr}\{P(\mathbf{S})>p\}$ are available, we can use $\mathcal{E}\left(\sigma^{2}, \nu^{2}\right)$ to bound the worst-case error probability.

Now suppose that we have a bound on a moment of $P(\mathbf{S})$. For concreteness, consider a constraint on the mean

$$
\mathbf{E}\{P(\mathbf{S})\} \triangleq \frac{1}{N L K} \sum_{i=0}^{N L K-1} \mathbf{E} S_{i}^{2} \leq E / N L \sigma^{2} .
$$

Let $\mathcal{F} \triangleq\left\{F: \int p d F(p) \leq E / N L \sigma^{2}\right\}$, i.e., the set of all distribution functions of $P(\mathbf{S})$ that satisfy (12). The worst-case error probability is then bounded by

$$
\begin{aligned}
\hat{\mathcal{E}}\left(\sigma^{2}, \nu^{2}\right) & \triangleq \sup _{\mathbf{S}} \mathbf{E}\left\{\varepsilon\left(\mathbf{S}, \nu^{2}\right)\right\} \\
& \leq \sup _{F \in \mathcal{F}} \int_{0}^{\infty} \mathcal{E}\left(E / N L p, \nu^{2}\right) d F(p)
\end{aligned}
$$

where the first supremum is over all $\mathbf{S}$ satisfying (12).

It is not difficult to see that many energy constraints can be treated in the same way as (12), by modifying $\mathcal{F}$ appropriately. Observe that the performance of the communication system for such problems is closely tied to its performance for the constraint (9). Moreover, any bound obtained on $\mathcal{E}\left(\sigma^{2}, \nu^{2}\right)$ can be translated into a bound on the worst-case error for other energy constraints through (11).

\section{AN UPPER BOUND TO $\mathcal{E}\left(\sigma^{2}, \nu^{2}\right)$}

A simple upper bound to $\mathcal{E}\left(\sigma^{2}, \nu^{2}\right)$ can be found by applying the union bound $[17$, p. 264] to (8), which yields

$$
\varepsilon\left(\mathbf{s}, \nu^{2}\right) \leq \frac{1}{M} \sum_{m=1}^{M} \sum_{\tilde{m} \neq m} \operatorname{Pr}\{m \rightarrow \tilde{m} \mid \mathbf{S}=\mathbf{s}\}
$$

where

$$
\operatorname{Pr}\{m \rightarrow \tilde{m} \mid \mathbf{S}=\mathbf{s}\} \triangleq \operatorname{Pr}\left\{\left\langle\mathbf{Y}, \mathbf{V}^{(m)}\right\rangle \leq\left\langle\mathbf{Y}, \mathbf{V}^{(\tilde{m})}\right\rangle \mid \mathbf{S}=\mathbf{s}\right\}
$$

and $\mathbf{Y}=\mathbf{V}^{(m)}+\mathbf{W}+\hat{\mathbf{S}}$. It then follows from (10) that

$$
\begin{aligned}
& \mathcal{E}\left(\sigma^{2}, \nu^{2}\right) \leq \frac{1}{M} \sum_{m=1}^{M} \sum_{\tilde{m} \neq m} \sup _{\mathbf{s}: P(\mathbf{s}) \leq E / N L \sigma^{2}} \\
& \cdot \operatorname{Pr}\{m \rightarrow \tilde{m} \mid \mathbf{S}=\mathbf{s}\} \text {. }
\end{aligned}
$$


Most of this section is devoted to finding bounds for the summands on the right. To begin, observe that

$$
\begin{aligned}
\operatorname{Pr}\{m \rightarrow \tilde{m} \mid \mathbf{S}=\mathbf{s}\} & \\
\triangleq & \operatorname{Pr}\left\{\left\langle\mathbf{Y}, \mathbf{V}^{(m)}\right\rangle \leq\left\langle\mathbf{Y}, \mathbf{V}^{(\tilde{m})}\right\rangle \mid \mathbf{S}=\mathbf{s}\right\} \\
=\operatorname{Pr} & \left\{\sum_{\ell=0}^{L-1} \sum_{n=0}^{N-1}\left(s_{J_{\ell} N+n}+W_{\ell, n}\right) A_{J_{\ell} N+n}\left[x_{\ell}^{(\tilde{m})}-x_{\ell}^{(m)}\right]\right. \\
& \left.\geq \sum_{\ell=0}^{L-1} \sum_{n=0}^{N-1} A_{J_{\ell} N+n}^{2} \sqrt{\frac{E}{L N}} x_{\ell}^{(m)}\left[x_{\ell}^{(m)}-x_{\ell}^{(\tilde{m})}\right]\right\} .
\end{aligned}
$$

Defining $d_{m \tilde{m}}$ to be the Hamming distance between the codewords $\mathbf{X}^{(m)}$ and $\mathbf{X}^{(\tilde{m})}$, the sum on the right side reduces to

$$
\sum_{\ell=0}^{L-1} \sum_{n=0}^{N-1} A_{J_{\ell} N+n}^{2} \sqrt{\frac{E}{L N}} x_{\ell}^{(m)}\left[x_{\ell}^{(m)}-x_{\ell}^{(\tilde{m})}\right]=2 d_{m \tilde{m}} \sqrt{\frac{N E}{L}} .
$$

Now define $b_{\ell} \triangleq\left[x_{\ell}^{(\tilde{m})}-x_{\ell}^{(m)}\right] / 2,0 \leq \ell<L$ and rewrite (15) as

$$
\begin{aligned}
\operatorname{Pr}\{m \rightarrow \tilde{m} \mid \mathbf{S}=\mathbf{s}\} \\
=\operatorname{Pr}\left\{\sum_{\ell=0}^{L-1} b_{\ell} \sum_{n=0}^{N-1} A_{J_{\ell} N+n}\left(s_{J_{\ell} N+n}+W_{\ell, n}\right)\right. \\
\left.\geq d_{m \tilde{m}} \sqrt{\frac{N E}{L}}\right\} \\
=\operatorname{Pr}\left\{\sum_{\ell=0}^{L-1} b_{\ell} \sum_{n=0}^{N-1} A_{J_{\ell} N+n} s_{J_{\ell} N+n}+\hat{W}\right. \\
\left.\geq d_{m \tilde{m}} \sqrt{\frac{N E}{L}}\right\}
\end{aligned}
$$

where $\hat{W} \triangleq \sum_{\ell=0}^{L-1} b_{\ell} \sum_{n=0}^{N-1} A_{J_{\ell}+n} W_{\ell, n}$ is a Gaussian random variable with mean zero and variance $N d_{m \tilde{m}} N_{0} / 2$, which is independent of the $J_{\ell}$ and $A_{i}$.

To proceed further, we apply the Chernoff bound [3, p. 134] to the probability on the right. For all $\lambda \geq 0$, we obtain

$$
\begin{aligned}
\operatorname{Pr}\{m \rightarrow \tilde{m} \mid \mathbf{S}=\mathbf{s}\} & \leq E_{\mathbf{A}, \mathbf{J}}\left\{\exp \left(\lambda \sum_{\ell=0}^{L-1} b_{\ell} \sum_{n=0}^{N-1} A_{J_{\ell} N+n} s_{J_{\ell} N+n}\right)\right\} \\
& \cdot E_{\hat{W}}\{\exp (\lambda \hat{W})\} \cdot \exp \left(-\lambda d_{m \tilde{m}} \sqrt{\frac{N E}{L}}\right) \\
= & E_{\mathbf{J}}\left\{E_{\mathbf{A}}\left[\exp \left(\lambda \sum_{\ell=0}^{L-1} b_{\ell} \sum_{n=0}^{N-1} A_{J_{\ell} N+n} s_{J_{\ell} N+n}\right) \mid \mathbf{J}\right]\right\} \\
& \cdot \exp \left(\lambda^{2} d_{m \tilde{m}} \frac{N N_{0}}{4}-\lambda d_{m \tilde{m}} \sqrt{\frac{N E}{L}}\right) \\
= & E_{\mathbf{J}}\left\{\prod_{\ell=0}^{L-1} \prod_{n=0}^{N-1} \cosh \left(\lambda s_{J_{\ell}} N+n b_{\ell}\right)\right\} \\
& \cdot \exp \left(\lambda^{2} d_{m \tilde{m}} \frac{N N_{0}}{4}-\lambda d_{m \tilde{m}} \sqrt{\frac{N E}{L}}\right)
\end{aligned}
$$

$$
\begin{gathered}
=E_{\mathbf{J}}\left\{\prod_{n=0}^{N-1} \prod_{\ell=0}^{L-1} \cosh \left(\lambda s_{J_{\ell} N+n}\left|b_{\ell}\right|\right)\right\} \\
\cdot \exp \left(\lambda^{2} d_{m \tilde{m}} \frac{N N_{0}}{4}-\lambda d_{m \tilde{m}} \sqrt{\frac{N E}{L}}\right)
\end{gathered}
$$

where $E_{\hat{W}}, E_{\mathbf{A}}$, and $E_{\mathbf{J}}$ denote expectations with respect to the distributions of $\hat{W}, \mathbf{A} \triangleq\left(A_{0}, \cdots, A_{K L N-1}\right)$, and $\mathbf{J} \triangleq\left(J_{0}, \cdots, J_{L-1}\right)$, respectively. Let $I$ be the set of $d_{m \tilde{m}}$ indexes $\ell$ such that $\left|b_{\ell}\right|=1$ and observe that $\left|b_{\ell}\right|=0$ for $\ell \notin I$. For all $0 \leq j<K L$, let $H_{j}\left(d_{m \tilde{m}}\right)=1$ if $j=J_{\ell}$ for some $\ell \in I$ and let $H_{j}\left(d_{m \tilde{m}}\right)=0$ otherwise. It follows from this definition that

$$
\prod_{j=0}^{K L-1} \cosh \left[\lambda s_{j N+n} H_{j}\left(d_{m \tilde{m}}\right)\right]=\prod_{\ell=0}^{L-1} \cosh \left(\lambda s_{J_{\ell} N+n}\left|b_{\ell}\right|\right) .
$$

Recalling that $\mathbf{J}$ is uniformly distributed over all permutations of size $L$ from $\{0,1, \cdots, K L-1\}$, we see that $\mathbf{H}\left(d_{m \tilde{m}}\right) \triangleq\left[H_{0}\left(d_{m \tilde{m}}\right), \cdots, H_{K L-1}\left(d_{m \tilde{m}}\right)\right]$ is uniformly distributed over all binary $\{0,1\} K L$-tuples with Hamming weight $d_{m \tilde{m}}$. More generally, it will be convenient to define

$$
\begin{aligned}
& \Phi(k) \triangleq E_{\mathbf{H}(k)}\left\{\prod_{j=0}^{K L-1} \prod_{n=0}^{N-1} \cosh \left[\lambda s_{j N+n} H_{j}(k)\right]\right\} \\
& 0 \leq k \leq K L
\end{aligned}
$$

where $\mathbf{H}(k) \triangleq\left[H_{0}(k), \cdots, H_{K L-1}(k)\right]$ is uniformly distributed over all binary $\{0,1\} K L$-tuples of weight $k$. Using (17), we can rewrite (16) as

$$
\begin{aligned}
& \operatorname{Pr}\{m \rightarrow \tilde{m} \mid \mathbf{S}=\mathbf{s}\} \\
& \quad \leq \Phi\left(d_{m \tilde{m}}\right) \cdot \exp \left(\lambda^{2} d_{m \tilde{m}} \frac{N N_{0}}{4}-\lambda d_{m \tilde{m}} \sqrt{\frac{N E}{L}}\right) .
\end{aligned}
$$

The factor $\Phi\left(d_{m \tilde{m}}\right)$ is generally difficult to reduce further. Here we develop a simple upper bound for $\Phi\left(d_{m \tilde{m}}\right)$ in terms of a similar expectation for Bernoulli random variables. Define

$$
\begin{aligned}
& \Psi(q) \triangleq E_{\mathbf{B}(q)}\left\{\prod_{j=0}^{K L-1} \prod_{n=0}^{N-1} \cosh \left[\left(\lambda s_{j N+n} B_{j}(q)\right]\right\}\right. \\
& 0 \leq q \leq 1
\end{aligned}
$$

where $\mathbf{B}(q) \triangleq\left[B_{0}(q), \cdots, B_{K L-1}(q)\right]$ is a $K L$-tuple of i.i.d. Bernoulli random variables with $\operatorname{Pr}\left\{B_{j}(q)=1\right\}=$ $1-\operatorname{Pr}\left\{B_{j}(q)=0\right\}=q$ for $0 \leq j<K L$. This quantity is easily evaluated as

$$
\begin{aligned}
\Psi(q) & =\prod_{j=0}^{K L-1} E_{B_{j}(q)}\left\{\prod_{n=0}^{N-1} \cosh \left[\lambda s_{j N+n} B_{j}(q)\right]\right\} \\
& =\prod_{j=0}^{K L-1}\left\{q \prod_{n=0}^{N-1} \cosh \left(\lambda s_{j N+n}\right)+1-q\right\}
\end{aligned}
$$

We can also express $\Psi(q)$ in terms of $\Phi(k)$ by conditioning on the Hamming weight of $\mathbf{B}(q)$. Letting $B \triangleq \sum_{j=0}^{K L-1} B_{j}(q)$, 
we obtain

$$
E_{\mathbf{B}(q)}\left\{\prod_{j=0}^{K L-1} \prod_{n=0}^{N-1} \cosh \left[\lambda s_{j N+n} B_{j}(q)\right] \mid B=k\right\}=\Phi(k)
$$

hence

$$
\begin{aligned}
\Psi(q) & =E_{B}\left\{E_{\mathbf{B}(q)}\left(\prod_{j=0}^{K L-1} \prod_{n=0}^{N-1} \cosh \left[\lambda s_{j N+n} B_{j}(q)\right] B\right)\right\} \\
& =\sum_{k=0}^{K L} \Phi(k)\left(\begin{array}{c}
K L \\
k
\end{array}\right) q^{k}(1-q)^{K L-k} .
\end{aligned}
$$

In the Appendix, we prove that $\Phi(k)$ is increasing in $k$. For any $0<p<1$, this implies

$$
\begin{aligned}
\sum_{k=d}^{d^{\prime}} \Phi(d)\left(\begin{array}{c}
K L \\
k
\end{array}\right) p^{k}(1-p)^{K L-k} & \\
& \leq \sum_{k=d}^{d^{\prime}} \Phi(k)\left(\begin{array}{c}
K L \\
k
\end{array}\right) p^{k}(1-p)^{K L-k}
\end{aligned}
$$

and hence

$$
\Phi(d) \leq \frac{\sum_{k=0}^{K L} g(k) \Phi(k)\left(\begin{array}{c}
K L \\
k
\end{array}\right) p^{k}(1-p)^{K L-k}}{\sum_{k=d}^{d^{\prime}}\left(\begin{array}{c}
K L \\
k
\end{array}\right) p^{k}(1-p)^{K L-k}}
$$

for all $d \leq d^{\prime} \leq K L$, where

$$
g(k) \triangleq \begin{cases}1, & d \leq k \leq d^{\prime} \\ 0, & \text { elsewhere. }\end{cases}
$$

Since $g(k) \leq \exp \left[-\rho\left(k-d^{\prime}\right)\right]$ for all $\rho \geq 0$, we set $p \triangleq d / K L$ and bound the numerator in (23) by

$$
\begin{aligned}
& \sum_{k=0}^{K L} \exp \left[-\rho\left(k-d^{\prime}\right)\right] \Phi(k)\left(\begin{array}{c}
K L \\
k
\end{array}\right) p^{k}(1-p)^{K L-k} \\
& =\exp \left[\rho\left(d^{\prime}-d\right)\right]\left[\left(\frac{p e^{-\rho}+1-p}{e^{-\rho}}\right)^{p}\right. \\
& \left.\cdot\left(p e^{-\rho}+1-p\right)^{1-p}\right]^{K L} \\
& \cdot \sum_{k=0}^{K L} \Phi(k)\left(\begin{array}{c}
K L \\
k
\end{array}\right)\left(\frac{p e^{-\rho}}{p e^{-\rho}+1-p}\right)^{k} \\
& \cdot\left(\frac{1-p}{p e^{-\rho}+1-p}\right)^{K L-k} \\
& =\exp \left[\rho\left(d^{\prime}-d\right)\right]\left[\left(\frac{p}{q}\right)^{p}\left(\frac{1-p}{1-q}\right)^{1-p}\right]^{K L} \Psi(q) \\
& =\exp \left[\rho\left(d^{\prime}-d\right)+K L D(p, q)\right] \Psi(q)
\end{aligned}
$$

where

$$
q \triangleq \frac{p e^{-\rho}}{p e^{-\rho}+1-p}
$$

and $D(p, q)$ is the Kullback-Leibler distance [3, p. 110]

$$
D(p, q) \triangleq p \ln \left(\frac{p}{q}\right)+(1-p) \ln \left(\frac{1-p}{1-q}\right) .
$$

Combining (19), (23), and (24), we obtain (27), shown at the bottom of the page, which holds for all $\lambda \geq 0, \rho \geq 0$, and $d_{m \tilde{m}} \leq d^{\prime} \leq K L$. Here $p \triangleq d_{m \tilde{m}} / K L$, and $q$ is related to $p$ by (25).

Recall that our objective was to bound the summands on the right side of (14). Such a bound can be obtained by maximizing (27) over all $\mathbf{s}$ satisfying $P(\mathbf{s}) \leq E / N L \sigma^{2}$. The only term in (27) that depends on $\mathbf{s}$ is $\Psi(q)$. Hence, the maximization problem at hand may be stated as [cf., (21)]

$$
\max _{\mathrm{s}} \prod_{j=0}^{K L-1}\left\{q \prod_{n=0}^{N-1} \cosh \left(\lambda s_{j N+n}\right)+1-q\right\}
$$

subject to

$$
\frac{1}{N L K} \sum_{j=0}^{K L-1} \sum_{n=0}^{N-1} s_{j N+n}^{2} \leq \tau
$$

where $\tau \triangleq E / N L \sigma^{2}$.

In [7, p. 1195], we showed that if

$$
w_{j}^{2} \triangleq(1 / N) \sum_{n=0}^{N-1} s_{j N+n}^{2}
$$

then

$$
\prod_{n=0}^{N-1} \cosh \left(\lambda s_{j N+n}\right) \leq \cosh ^{N}\left(\lambda w_{j}\right)
$$

with equality if and only if $s_{j N+n}^{2}=w_{j}^{2}, 0 \leq n<N$. Thus, (28) reduces to

$$
\max _{\mathbf{w}} \prod_{j=0}^{K L-1}\left\{q \cosh ^{N}\left(\lambda w_{j}\right)+1-q\right\}
$$

subject to

$$
\frac{1}{K L} \sum_{j=0}^{K L-1} w_{j}^{2} \leq \tau .
$$

The solution to this problem is more easily seen if we set $u_{j} \triangleq \lambda^{2} w_{j}^{2}$ and rewrite the problem in the alternate form

$$
\max _{\mathbf{u}} \frac{1}{K L} \sum_{j=0}^{K L-1} h\left(u_{j}\right)
$$

subject to

$$
\frac{1}{K L} \sum_{j=0}^{K L-1} u_{j} \leq \tau \lambda^{2}, \quad u_{j} \geq 0
$$

$$
\operatorname{Pr}\{m \rightarrow \tilde{m} \mid \mathbf{S}=\mathbf{s}\} \leq \frac{\exp \left[\rho\left(d^{\prime}-d_{m \tilde{m}}\right)+K L D(p, q)+\lambda^{2} d_{m \tilde{m}} N N_{0} / 4-\lambda d_{m \tilde{m}} \sqrt{N E / L}\right] \cdot \Psi(q)}{\sum_{k=d_{m \tilde{m}}}^{d^{\prime}}\left(\begin{array}{c}
K L \\
k
\end{array}\right) p^{k}(1-p)^{K L-k}}
$$




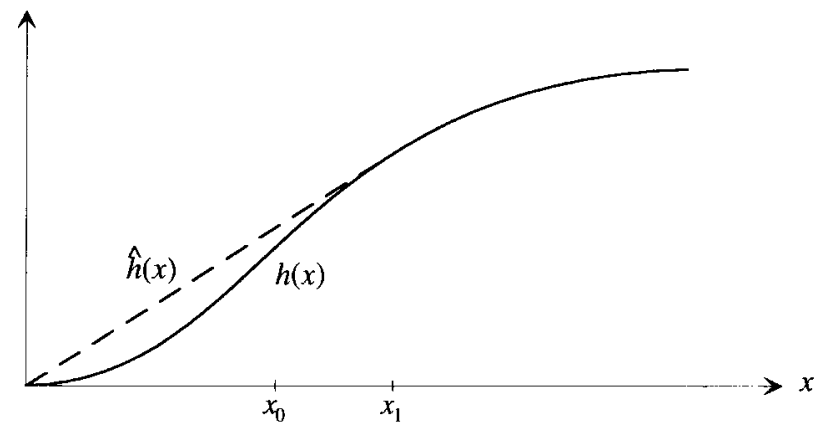

Fig. 3. Behavior of $\hat{h}(x)$ and $h(x)$ for $q<(3 N-2) / 3 N$.

where

$$
h(x) \triangleq \ln \left[q \cosh ^{N}(\sqrt{x})+1-q\right], \quad x \geq 0
$$

so that

$$
\begin{aligned}
\max _{\mathbf{s}} \prod_{j=0}^{K L-1}\left\{q \prod_{n=0}^{N-1} \cosh \left(\lambda s_{j N+n}\right)\right. & +1-q\} \\
& =\exp \left[\max _{\mathbf{u}} \sum_{j=0}^{K L-1} h\left(u_{j}\right)\right] .
\end{aligned}
$$

Observe that $h(x)$ can be bounded above by a function derived from a two-point interpolation of $h(x)$

$$
\begin{aligned}
\hat{h}(x) & \triangleq \sup _{0<\alpha \leq 1}(1-\alpha) h(0)+\alpha h(x / \alpha) \\
& =\sup _{0<\alpha \leq 1} \alpha h(x / \alpha) \\
& \geq h(x) .
\end{aligned}
$$

In the Appendix, we show that $h(x)$ is a strictly increasing function which is: 1) concave for all $x>0$ if $q>(3 N-$ 2) $/ 3 N$ and 2) convex for $x<x_{0}$ and concave for $x \geq x_{0}$ if $q \leq(3 N-2) / 3 N$ (where $x_{0}$ depends upon $q$ ). When $h(x)$ is concave for all $x>0$, we have $\hat{h}(x)=h(x)$ for all $x \geq 0$. Otherwise, as illustrated in Fig. 3, it can be shown (see [17, App. 7b]) that, for some $x_{1}>x_{0}, \hat{h}(x)$ consists of a straight line for all $x<x_{1}$, which is tangent to $h(x)$ at $x_{1}$, and $\hat{h}(x)=h(x)$ for $x \geq x_{1}$. In either case, $\hat{h}(x)$ is concave and increasing for all $x \geq 0$.

Since $\hat{h}(x)$ is concave, increasing, and $\hat{h}(x) \geq h(x)$, it follows that

$$
\begin{aligned}
\frac{1}{K L} \sum_{j=0}^{K L-1} h\left(u_{j}\right) & \leq \frac{1}{K L} \sum_{j=0}^{K L-1} \hat{h}\left(u_{j}\right) \\
& \leq \hat{h}\left(\frac{1}{K L} \sum_{j=0}^{K L-1} u_{j}\right) \\
& \leq \hat{h}\left(\tau \lambda^{2}\right) .
\end{aligned}
$$

Hence, we have established that

$$
\max _{\mathbf{s}: P(\mathbf{s}) \leq \tau} \Psi(q) \leq \exp \left[K L \hat{h}\left(\tau \lambda^{2}\right)\right] .
$$

Equality in this bound is approached as $K L$ becomes large.

Since (27) is valid for any $\lambda \geq 0$, we may replace $\lambda$ everywhere by $\lambda / \sqrt{\tau}$. Combining (27) and (34), we then obtain

$$
\begin{aligned}
& \sup _{\mathbf{s :}} \quad \operatorname{P(s)\leq E/NL\sigma ^{2}} \operatorname{Pr}\{m \rightarrow \tilde{m} \mid \mathbf{S}=\mathbf{s}\} \leq \\
& \sup _{0<\alpha \leq 1} \frac{\exp \left[\rho\left(d^{\prime}-d_{m \tilde{m}}\right)-K L E\left(p, N, \sigma^{2}, \nu^{2}, \alpha, \lambda, q\right)\right]}{\sum_{k=d_{m \tilde{m}}}^{d^{\prime}}\left(\begin{array}{c}
K L \\
k
\end{array}\right) p^{k}(1-p)^{K L-k}}
\end{aligned}
$$

for all $d_{m \tilde{m}} \leq d^{\prime} \leq K L, \lambda \geq 0, \rho \geq 0$, where

$$
\begin{aligned}
E\left(p, N, \sigma^{2}, \nu^{2}, \alpha, \lambda, q\right) \triangleq & \lambda p N \sigma-D(p, q)-\frac{p N \lambda^{2} \sigma^{2}}{2 \nu^{2}} \\
& -\alpha h\left(\lambda^{2} / \alpha\right) .
\end{aligned}
$$

Here $q, D(p, q)$, and $h(x)$ are as defined in (25), (26), and (31), respectively.

The tightest bound is obtained by minimizing with respect to the variables in (35). Doing so, we obtain the main result of this section

$$
\mathcal{E}\left(\sigma^{2}, \nu^{2}\right) \leq \frac{1}{M} \sum_{m=1}^{M} \sum_{\tilde{m} \neq m} \beta\left(d_{m \tilde{m}}\right)
$$

where

$$
\beta(d) \triangleq \min _{d \leq d^{\prime} \leq K L} \frac{\exp \left[\rho_{0}\left(d^{\prime}-d\right)-K L E_{\mathrm{DS}}\left(p, N, \sigma^{2}, \nu^{2}\right)\right]}{\sum_{k=d}^{d^{\prime}}\left(\begin{array}{c}
K L \\
k
\end{array}\right) p^{k}(1-p)^{K L-k}}
$$$$
p \triangleq d / K L, \nu^{2} \triangleq 2 E / N L N_{0}, \sigma^{2} \triangleq E / P_{I} T \text {, and }
$$

$E_{\mathrm{DS}}\left(p, N, \sigma^{2}, \nu^{2}\right)$

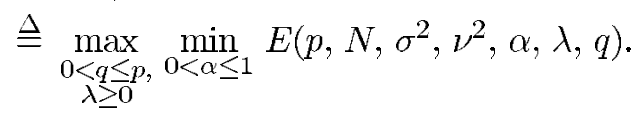

Here, $\rho_{0}$ is obtained from $p$ and the optimizing $q$ through (25). We show in [6, pp. 64-73] that the optimization in (39) can be accomplished through the simultaneous solution of the equations satisfying the first-order necessary conditions for $\alpha$, $\lambda$, and $q$, and that $E_{\mathrm{DS}}\left(p, 1, \sigma^{2}, \nu^{2}\right)$ is the true asymptotic exponent in $L$ of the error probability. Thus, the bound of (37) is asymptotically tight as $L$ becomes large for $N=1$. The methods of [6] can be used with minor changes to prove asymptotic tightness of (37) for arbitrary $N$.

A simpler bound can be obtained using the minimum Hamming distance $\bar{d}$ of the code

$$
\begin{aligned}
\mathcal{E}\left(\sigma^{2}, \nu^{2}\right) \leq & (M-1) \beta(\bar{d}) \\
\leq & \frac{(M-1) \exp \left[-K L E_{\mathrm{DS}}\left(p, N, \sigma^{2}, \nu^{2}\right)\right]}{\left(\begin{array}{c}
K L \\
\bar{d}
\end{array}\right) p^{\bar{d}}(1-p)^{K L-\bar{d}}} \\
\leq & (M-1) \sqrt{8 K L p(1-p)} \\
& \cdot \exp \left[-K L E_{\mathrm{DS}}\left(p, N, \sigma^{2}, \nu^{2}\right)\right]
\end{aligned}
$$

where $p \triangleq \bar{d} / K L$. Here, the second inequality follows by setting $d^{\prime}=\bar{d}$ and the third by $\left(\frac{K L}{d}\right) p^{\bar{d}}(1-p)^{K L-\bar{d}} \geq$ $1 / \sqrt{8 K L p(1-p)}$, which is a consequence of Stirling's approximation (e.g., [3, p. 18]). Although (40) is slightly looser than (37), it is still asymptotically tight as $K L \rightarrow+\infty$. 


\section{DISCUSSION}

In Section III, we derived an upper bound to the worstcase error probability. In this section, we identify the type of interference that maximizes this bound and explore the dependence of this bound on the choice of system parameters.

\section{A. Worst-Case Interference}

It is difficult to determine the interference that maximizes the error probability $\mathcal{E}\left(\sigma^{2}, \nu^{2}\right)$; however, we can identify the type of interference that maximizes the upper bound (35). By (29), the worst-case $\mathbf{s}$ has a constant magnitude on bit intervals, so that $s_{j N+n}^{2}=w_{j}^{2}, 0 \leq n<N$. Moreover, to achieve equality in (33), $w_{j}^{2}$ should take on only two values, 0 and $E \lambda_{o}^{2} / N L \sigma^{2} \alpha_{o}$, where $\lambda_{o}$ and $\alpha_{o}$ optimize the exponent in (39). It follows that $S(t)$ is of the form

$$
\begin{aligned}
S_{D}(t) \triangleq & \sum_{j=0}^{K L-1} Z_{j} \sum_{n=0}^{N-1} \sqrt{\frac{2 P_{I} K L}{D}} \cos (\omega t) \\
& \cdot v\left[t-(j N+n) T_{C}\right], \quad 0 \leq t<K T
\end{aligned}
$$

for some integer $1 \leq D \leq K L$, where $\left(Z_{0}, \cdots, Z_{K L-1}\right)$ is any binary sequence with Hamming weight $D$. Here, the amplitude of the signal is chosen so that $S_{D}(t)$ has the maximum energy permitted by the constraint (4), i.e., $\int_{0}^{K T} S_{D}^{2}(t) d t=K T P_{I}$. Thus, the worst upper bound on the error probability is inflicted by an interference that is synchronized and in-phase with the transmitter, uses the same chip waveform, and is pulsed on bit intervals with a duty factor which depends on $\sigma^{2}$ and $\nu^{2}$.

Some feeling for the tightness of the upper bound can be gained by comparing it to the error probability caused by the interference $S_{D}(t)$. Fig. 4 plots the upper bound (37) for a biorthogonal code with $(L, M, \bar{d})=(32,64,16)$ [4, p. 333], $K=5, N=32$, and $\nu^{2}=\infty$ (no thermal noise) versus the bit-energy-to-interference-density ratio

$$
\frac{E_{b}}{N_{I}} \triangleq \frac{E}{P_{I} T} \cdot \frac{W}{R}=\frac{N L \sigma^{2}}{\log _{2} M}
$$

where $W=1 / T_{C}$ is the system bandwidth and $R=$ $(1 / T) \log _{2} M$ is the data rate. Also shown is a lower bound on the worst-case error probability, obtained by simulating the exact error probability (8) for $S_{D}(t)$ and maximizing with respect to $D$. For large $\sigma^{2}$, the maximum usually occurs at $D=1$; consequently, the exact error probability for $D=1$ is also shown in Fig. 4 for comparison. For large $E_{b} / N_{I}$, the figure shows that the upper bound is within $2 \mathrm{~dB}$ of the worstcase error probability; for small $E_{b} / N_{I}$, however, the bounds are far apart and the figure admits no conclusion.

As mentioned in Section II, all of the results in this paper are easily adapted to QPSK, which can be viewed as two quadrature-multiplexed BPSK signals. With QPSK, binary data can be transmitted in half the time taken by BPSK. If we hold average transmitter and interference power fixed, the upper bound (35) still holds with $E$ and $T$ replaced by $E / 2$ and $T / 2$, respectively. This is equivalent to halving the signalto-noise ratio $\nu^{2}$ and doubling the data rate $R$, with all other

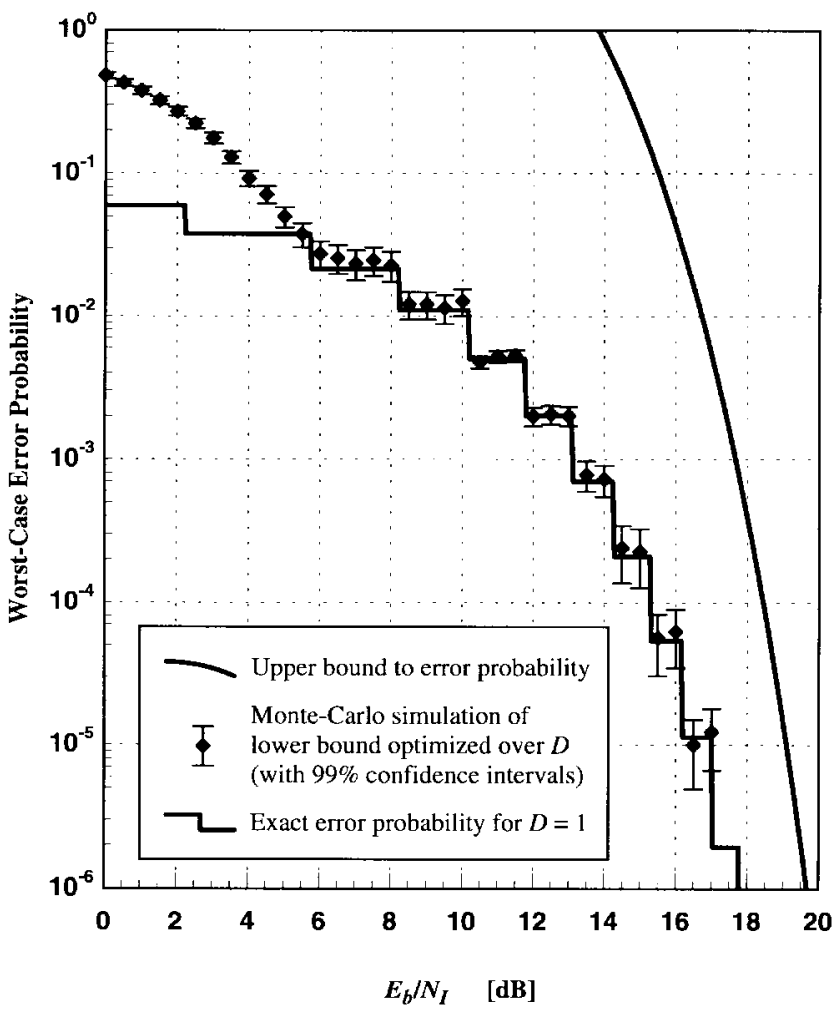

Fig. 4. Bounds on worst-case error probability for the $(32,64,16)$ biorthogonal code with $N=32, K=5$, and $\nu^{2}=\infty$.

parameters held fixed. The worst-case interference then takes a form similar to (41), but with sine as well as cosine terms.

\section{B. Symbol-Level Versus Chip-Level Interleaving}

The model given in Section II assumes interleaving at the code symbol level. It is interesting, however, to consider an alternate system in which interleaving is performed at the chip level (setting aside for a moment the practical difficulties which this entails). The worst-case performance of such a system can be obtained directly from (37) or (40) in the following way. First, consider a system $\mathcal{S}$ with parameters $L, N$, and $p$. Next, consider a system $\mathcal{S}^{\prime}$ with parameters $L^{\prime}=L N, N^{\prime}=1$, and $p^{\prime}=p$, where the code for system $\mathcal{S}^{\prime}$ is obtained by repeating $N$ times each symbol in the code for $\mathcal{S}$. Clearly, symbollevel interleaving of system $\mathcal{S}^{\prime}$ is equivalent to chip-level interleaving of system $\mathcal{S}$. Hence, we can write the exponent of the error bound for chip-level interleaving in terms of that for symbol-level interleaving

$$
E_{\mathrm{DS}}^{\text {chip }}\left(p, N, \sigma^{2}, \nu^{2}\right)=N E_{\mathrm{DS}}\left(p, 1, \sigma^{2}, \nu^{2}\right) .
$$

Equation (43) suggests that any change in $N$ will result in a proportional change in the error exponent when chip-level interleaving is used. Therefore, increasing the number of chips per symbol is equivalent to increasing the block length of the code (with $p$ fixed) when interleaving is performed at the chip level.

We now examine the exponents $E_{\mathrm{DS}}$ and $E_{\mathrm{DS}}^{\mathrm{chip}}$ for the (32, $64,16)$ biorthogonal code with $K=5$. Since the exponents in (39) and (43) depend on $(L, M, \bar{d})$ only through the ratio $p \triangleq \bar{d} / K L$, the exponents for any other orthogonal 


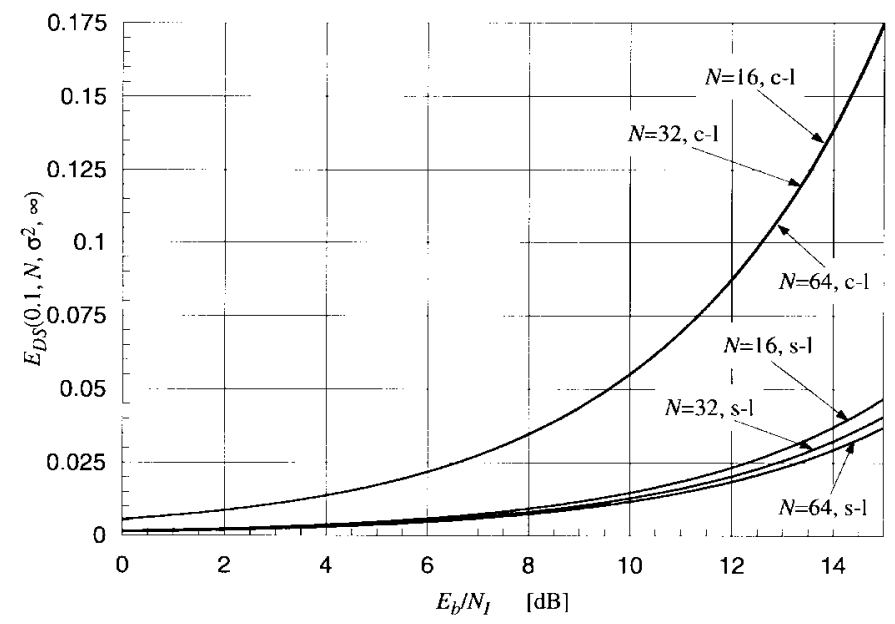

Fig. 5. Comparison of symbol-level and chip-level interleaving.

or biorthogonal code with parameters $\left(L^{\prime}, M^{\prime}, \bar{d}^{\prime}\right)$ can be obtained by shifting the $(L, M, \bar{d})$ curves to the right by $10 \log _{10}\left(L \log _{2} M^{\prime} / L^{\prime} \log _{2} M\right)$ decibels.

In Fig. 5 , we plot the error exponents $E_{\mathrm{DS}}$ (labeled $s-l$ ) and $E_{\mathrm{DS}}^{\text {chip }}$ (labeled $c-l$ ) for $N=16,32,64$ in the absence of thermal noise $\left(\nu^{2}=\infty\right)$. Note that, when thermal noise is absent, both exponents become unbounded for $p \sigma^{2}>1$, which corresponds to a worst-case error probability of zero. We observe from Fig. 5 that these exponents do not vary significantly with $N$. We also see that the exponents for chip-level interleaving $(c-l)$ are roughly $6 \mathrm{~dB}$ better than the corresponding exponents for symbol-level interleaving $(s-l)$. The gap between these curves widens as $K$ increases. This is because, as $K$ increases, $p$ decreases and so does the worstcase $\alpha$ in (39). A small $\alpha$ corresponds to a pulsed interfering signal [cf., (34)], which inflicts a large error probability (and hence a small exponent) on the correlation receiver for symbol interleaving. However, the chip interleaving system is more robust to pulsed interference, and its exponent is not reduced as much. This difference suggests that interleaving below the symbol level improves worst-case performance. While chiplevel interleaving may be difficult to achieve in practice for large $N$, our results suggest that performance can be improved by interleaving on any time scale finer than the symbol duration.

\section{Thermal Noise Versus Interference}

In order to compare the effects of thermal noise with arbitrary interference, we define the composite signal-to-noise ratio to be $\sigma_{c}^{2} \triangleq E /\left(P_{I} T+N L N_{0} / 2\right)$ and let $\gamma \triangleq \sigma_{c}^{2} / \nu^{2}$, so that

$$
\sigma_{c}^{2}=\gamma \nu^{2}=(1-\gamma) \sigma^{2} .
$$

Hence, as $\gamma$ varies from 0 to 1 , the channel changes from one with only unknown interference to one with only thermal noise, while the total average noise power remains fixed.

In Fig. 6, we plot $E_{\mathrm{DS}}$ and $E_{\mathrm{DS}}^{\text {chip }}$ for $K=5, N=32$, the $(32,64,16)$ biorthogonal code, and various values of $\gamma$. The ordinate of these plots is $E_{b} / N_{c}$, which is defined as in (42) with $\sigma_{c}^{2}$ replacing $\sigma^{2}$. We see from Fig. 6 that the difference

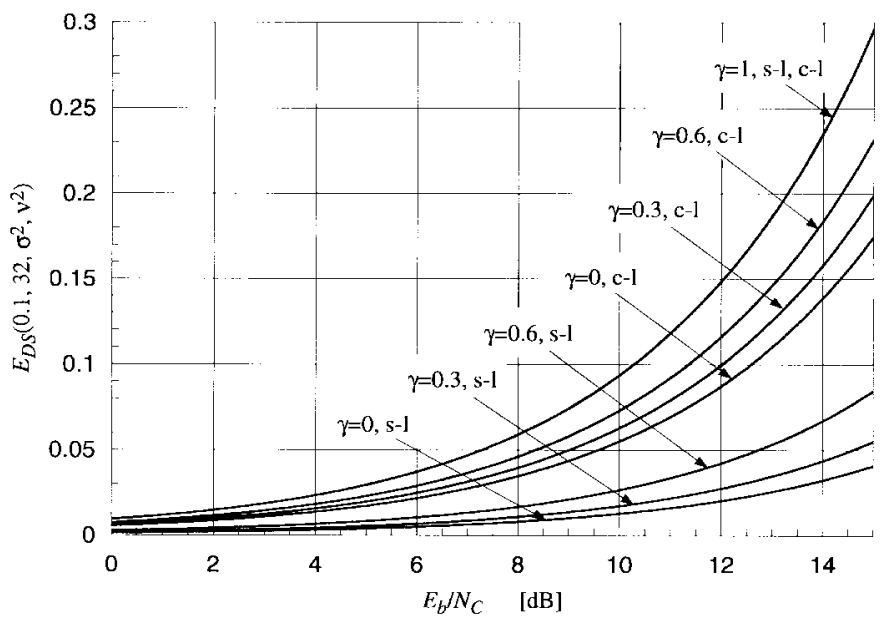

Fig. 6. Comparison of thermal noise with arbitrary interference.

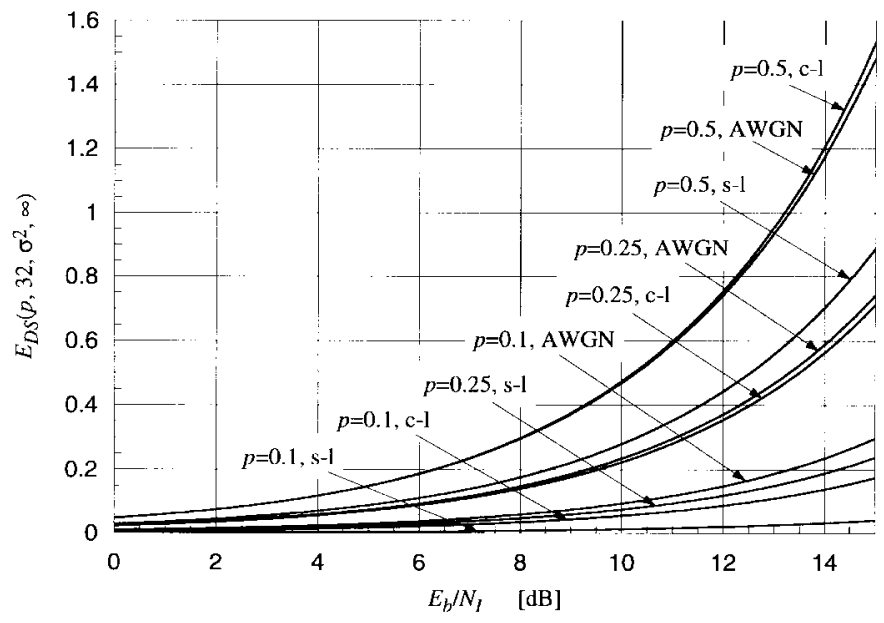

Fig. 7. Dependence on $p=\bar{d} / K L$.

between symbol-level and chip-level interleaving becomes less significant as the interference becomes less "unknown" and more "thermal." Indeed, these curves coincide for $\gamma=1$ but are separated by more than $4 \mathrm{~dB}$ for $\gamma \leq 0.6$. This is to be expected since interleaving has no effect on an additive white Gaussian noise (AWGN) channel. In the absence of unknown interference $(\gamma=1)$, both exponents simplify to the AWGN exponent

$$
E_{\mathrm{DS}}^{\mathrm{chip}}\left(p, N, \infty, \nu^{2}\right)=E_{\mathrm{DS}}\left(p, N, \infty, \nu^{2}\right)=\frac{N p \nu^{2}}{2} .
$$

\section{Dependence on $K$ and $\bar{d}$}

In Fig. 7, we plot $E_{\mathrm{DS}}$ and $E_{\mathrm{DS}}^{\text {chip }}$ for $N=32$, the (32, 64, 16) biorthogonal code, and $K=1,2,5(p=0.5,0.25,0.1)$ in the absence of thermal noise. For reference, we also plot the exponent of the AWGN channel with the same noise power (44).

As expected, we see that the exponents increase with $p$ (decrease with $K$ ). For small $p$, chip-level interleaving leads to substantially worse performance on the arbitrary interference channel than on the AWGN channel. However, the opposite is true for large $p$. This trend applies to chip-level interleaving 
for any $N$, since both the chip-level exponent (43) and the AWGN exponent (44) are linear in $N$. Fig. 7 also shows that the symbol-level exponents are worse on the arbitrary interference channel than on the AWGN channel for all $p$. Moreover, the performance of both types of interleaving on the arbitrary interference channel degrades relative to performance on the AWGN channel as $K$ increases. It is noteworthy that the same would be true if $K$ were fixed and $\bar{d}$ were decreased, since this would also decrease $p$.

This degradation in performance as $K$ increases is an artifact of the power constraint (4) and should not be construed as suggesting that increasing $K$ in a practical communication system would result in a higher error probability. Equation (41) suggests that worst-case interference is pulsed, and it is well known that the correlation receiver is not very effective in suppressing this type of interference. A smaller $K$ limits the ability of the interference to produce a high peak power, since any particular codeword can receive at most $K$ times the average interference power $P_{I}$.

\section{E. Asymptotically Optimal Random Modem and Detector}

In an earlier paper [8] we derived, for a fixed encoder, a random modem and detector that asymptotically minimize the worst-case error probability suffered on the channel (2) as the block length of the encoder becomes large. The feasible class of modems and detectors considered includes the DS system described in this paper. Thus, by comparing the performance of these two systems, we can determine how the performance of the DS system described in Section II compares with the optimally robust random modem and detector. In the absence of thermal noise $\left(N_{0}=0\right)$, the error exponent for the optimal random modem and detector on channel (2) reduces to [8]

$$
E_{o p t}\left(p, N, \sigma^{2}, \infty\right)=-\frac{N}{2} \ln \left(1-p \sigma^{2}\right) .
$$

In Fig. 8, we plot $E_{\text {opt }}, E_{\mathrm{DS}}$, and $E_{\mathrm{DS}}^{\text {chip }}$ under the same conditions as in Fig. 7. In this figure, the symbol-level exponents differ from the optimal exponents by more than 2,4 , and $6 \mathrm{~dB}$ for $p=0.5,0.25$, and 0.1 , respectively. In fact, these differences become more pronounced as $N$ is increased. This is another consequence of the pulsed nature of the worst-case noise.

The exponents for chip-level interleaving are close to optimal random modulation and detection for $p=0.5$. However, for smaller $p$, they are markedly suboptimal. This trend also holds for any $N$, since both the chip-level exponent (43) and the optimal exponent (45) are proportional to $N$.

\section{CONCLUSIONS}

In this paper, we considered a communication channel corrupted by Gaussian noise and by an arbitrary interfering signal with a strict bound on energy (4). For this channel, we derived an upper bound to the worst-case error probability of a DS spread-spectrum system with error-control coding, pseudorandom interleaving, and a correlation receiver. We further showed that this result can be used to bound the error probability even when (4) is violated, provided a bound on

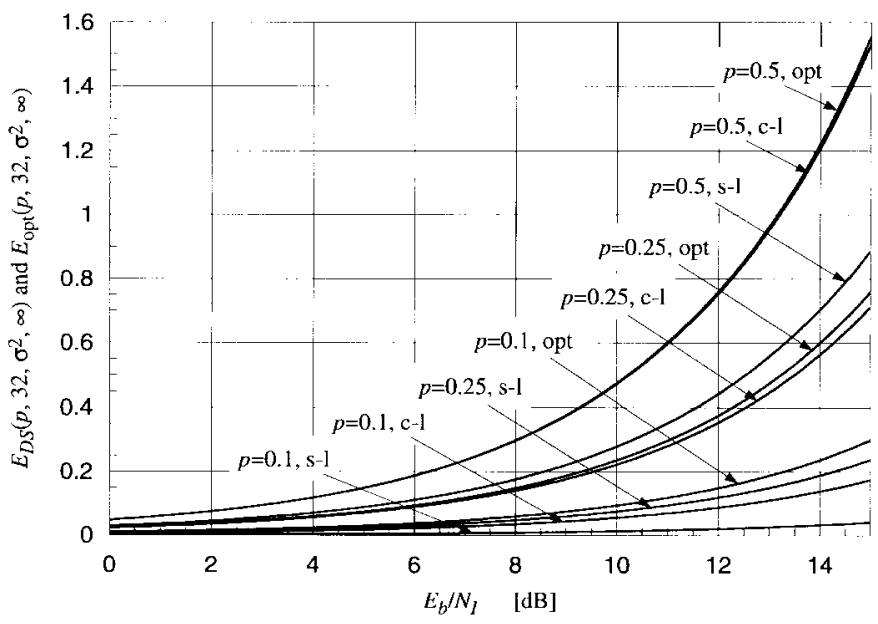

Fig. 8. Comparison of performance with optimal performance.

the tail probability or a moment of the interference energy is available.

The worst-case interference (in the sense of maximizing the upper bound) consists of a signal that is synchronized and in-phase with the transmitter, uses the same chip waveform, and is pulsed on symbol intervals with a duty factor that depends on the signal-to-interference and signal-to-noise power ratios $\sigma^{2}$ and $\nu^{2}$. Numerical examples suggest that, as the number of interleaved code symbols $K L$ becomes large compared to the minimum distance of the code $\bar{d}$, the duty factor becomes smaller, the worst-case interference becomes increasingly impulsive, and the error probability degrades dramatically. This performance degradation is to be expected, since correlation receivers are not very effective in suppressing impulsive interference.

Finally, we showed that the worst-case error probability can be improved by interleaving chips (or groups of chips) rather than bits, especially when the arbitrary interference dominates the Gaussian channel noise. While difficult to implement in practice for large $N$, chip-level interleaving leads to a communication system that is more robust to impulsive interference. We also compared the performance of both chiplevel and symbol-level interleaving to that of the modem and detector derived in [8], which asymptotically minimize the worst-case error probability as the code block length $L$ becomes large. For $K L \leq 2 \bar{d}$, the performance of chiplevel interleaving is nearly optimum; for $K L \gg \bar{d}$, however, performance is markedly suboptimum. In the latter case, performance can be significantly improved by using different modulation and detection. In particular, examination of the literature (e.g., [2], [16]) suggests that nonlinear detection would significantly improve the worst-case performance of the communication system considered here.

\section{APPENDIX}

Behavior of $\Phi(k)$ : We now show that $\Phi(k)$, defined in (18), is an increasing function of $k$. First, note that the random vector $\mathbf{H}(k)$ can be viewed as derived from $\mathbf{H}(k-1)$ by randomly choosing one of the $K L-k+1$ " 0 "s of $\mathbf{H}(k-1)$ 
and changing it into a " 1 ." Thus, we can rewrite (18) as

$$
\begin{gathered}
\Phi(k)=E_{\mathbf{H}(k-1)}\left(E _ { \mathbf { H } ( k ) } \left\{\prod_{j=0}^{K L-1} \prod_{n=0}^{N-1} \cosh \left[\lambda s_{j N+n} H_{j}(k)\right] \mid\right.\right. \\
\mathbf{H}(k-1)\}) \\
=E_{\mathbf{H}(k-1)}\left\{\prod_{j=0}^{K L-1} \prod_{n=0}^{N-1} \cosh \left[\lambda s_{j N+n} H_{j}(k-1)\right]\right. \\
\left.\sum_{j: H_{j}(k-1)=0}^{N-1} \cosh \left(\lambda s_{j N+n}\right) \frac{1}{K L-k+1}\right\}
\end{gathered}
$$

The summation on the right can be bounded below by

$$
\begin{aligned}
& \sum_{j: H_{j}(k-1)=0} \prod_{n=0}^{N-1} \cosh \left(\lambda s_{j N+n}\right) \frac{1}{K L-k+1} \\
& \geq \sum_{j: H_{j}(k-1)=0} \frac{1}{K L-k+1} \\
& =1 .
\end{aligned}
$$

Hence, we conclude that

$$
\begin{aligned}
\Phi(k) & \geq E_{\mathbf{H}(k-1)}\left\{\prod_{j=0}^{K L-1} \prod_{n=0}^{N-1} \cosh \left[\lambda s_{j N+n} H_{j}(k-1)\right]\right\} \\
& =\Phi(k-1)
\end{aligned}
$$

Behavior of $h(x)$ : Next we investigate the behavior of the function $h(x)$, defined in (31). For notational simplicity, we will write

$$
\begin{aligned}
& c \triangleq \cosh (\sqrt{x}) \\
& s \triangleq \sinh (\sqrt{x}) .
\end{aligned}
$$

First, observe that

$$
\frac{d h(x)}{d x}=\frac{q N c^{N-1} s}{2 \sqrt{x}\left(q c^{N}+1-q\right)}>0 \quad \text { for } x>0
$$

so $h(x)$ is strictly increasing.

Next, we examine the second derivative of $h(x)$

$$
\begin{aligned}
\frac{d^{2} h(x)}{d x^{2}}= & \frac{N q c^{N-2}}{4 x^{3 / 2}\left(q c^{N}+1-q\right)^{2}}\left[\left(q c^{N}+1-q\right)\left(N s^{2}+1\right)\right. \\
& \left.\cdot \sqrt{x}-c s\left(\sqrt{x} q N c^{N-1} s+q c^{N}+1-q\right)\right] . \quad(47)
\end{aligned}
$$

Since the fraction in (47) is positive for $x>0$, we only need to consider the term in square brackets to determine the concavity or convexity of $h(x)$. By a simple algebraic manipulation of this term, we find that $h(x)$ is concave if $q>g(x)$ and convex if $q<g(x)$ where

$$
g(x) \triangleq\left[1+\frac{c^{N}(c s-\sqrt{x})}{(N-1) \sqrt{x} s^{2}+c^{2}(\sqrt{x}-s / c)}\right]^{-1} .
$$

Since $c>1, s>\sqrt{x}$, and $s / c<\sqrt{x}$ for $x>0$, it is easily seen that $g(x)>0$ for $x>0$.
We now show that $g(x)$ is strictly decreasing. To see this, observe that

$$
\begin{aligned}
\frac{d g(x)}{d x}= & \frac{-g(x)^{2} N c^{N-1} s}{2 \sqrt{x}\left[(N-1) \sqrt{x} s^{2}+c^{2}(\sqrt{x}-s / c)\right]^{2}} \\
& \cdot\left[\sqrt{x} N c s^{3}+\sqrt{x} c s+(2-N) x s^{2}-2 s^{2}\right. \\
& \left.-2 s^{4}+x\right] .
\end{aligned}
$$

The fraction above is clearly negative for $x>0$. The term in square brackets reduces to

$$
\begin{aligned}
& \frac{N \sqrt{x}}{8} \sinh (4 \sqrt{x})-\frac{1}{4} \cosh (4 \sqrt{x})+\frac{(2-N) \sqrt{x}}{4} \\
& \cdot \sinh (2 \sqrt{x})+\frac{(2-N) x}{2} \cosh (2 \sqrt{x})+\frac{2 N x+1}{4}
\end{aligned}
$$

which can be expressed in a series expansion as

$$
\frac{1}{16} \sum_{i=3}^{\infty} \frac{(4 x)^{i}}{(2 i) !}\left[\left(4^{i} i-8 i^{2}\right) N-4^{i+1}+16 i^{2}\right] .
$$

It is easily shown that the bracketed expression in (48) takes on a minimum value of eight for all $N \geq 1$ and $i \geq 3$, which is attained by $N=1$ and $i=3$. Thus, each term in (48) is positive and, hence, $d g(x) / d x<0$ for $x>0$.

Since $g(x)$ is strictly decreasing, we have

and

$$
\begin{aligned}
& \sup _{x>0} g(x)=\lim _{x \rightarrow 0^{+}} g(x)=\frac{3 N-2}{3 N} \\
& \inf _{x>0} g(x)=\lim _{x \rightarrow+\infty} g(x)=0 .
\end{aligned}
$$

Recalling that $h(x)$ is concave if $q>g(x)$ and convex if $q<g(x)$, we conclude that if $q>(3 N-2) / 3 N, h(x)$ is concave for all $x>0$; otherwise, $h(x)$ is convex for $x<x_{0} \triangleq g^{-1}(q)$ and concave for $x>x_{0}$.

\section{REFERENCES}

[1] B. Aazhang and H. V. Poor, "Performance of DS/SSMA communications in impulsive channels-Part I: Linear correlation receivers," IEEE Trans. Commun., vol. 35, pp. 1179-1188, Nov. 1987.

[2] _ "Performance of DS/SSMA communications in impulsive channels-Part II: Hard-limiting correlation receivers," IEEE Trans. Commun., vol. 36, pp. 88-97, Jan. 1988.

[3] R. E. Blahut, Principles and Practice of Information Theory. Reading, MA: Addison-Wesley, 1987.

[4] G. C. Clark, Jr. and J. B. Cain, Error-Correction Coding for Digital Communications. New York: Plenum, 1981.

[5] R.-H. Dou and L. B. Milstein, "Error probability bounds and approximations for DS spread-spectrum communication systems with multiple-tone or multiple-access interference," IEEE Trans. Commun., vol. 32, pp. 493-502, May 1984.

[6] M. Hizlan, "Optimal random modulation for robust communication," Ph.D. dissertation, Johns Hopkins Univ., Baltimore, MD, 1990.

[7] M. Hizlan and B. L. Hughes, "On the optimality of direct-sequence for arbitrary interference rejection," IEEE Trans. Commun., vol. 39, pp. 1193-1196, Aug. 1991.

[8] B. L. Hughes and M. Hizlan, "An asymptotically optimal random modem and detector for robust communication," IEEE Trans. Inform. Theory, vol. 36, pp. 810-821, July 1990.

[9] R. Iltis and L. B. Milstein, "Performance analysis of narrow-band interference rejection techniques in DS spread-spectrum systems," IEEE Trans. Commun., vol. COM-32, pp. 1169-1177, Nov. 1984.

[10] P. A. Kullstam, "Spread spectrum performance analysis in arbitrary interference," IEEE Trans. Commun., vol. COM-25, pp. 848-853, Aug. 1977. 
[11] B. K. Levitt, "Effect of modulation format and jamming spectrum on performance of direct sequence spread spectrum systems," in Rec. Nat. Telecommunications Conf., vol. 1, Nov. 1980, pp. 3.4.1-3.4.5.

[12] L.-M. Li and L. B. Milstein, "Rejection of narrow-band interference in PN spread-spectrum systems using transversal filters," IEEE Trans. Commun., vol. 30, pp. 925-928, May 1982.

[13] L. B. Milstein, S. Davidovici, and D. L. Schilling, "The effect of multiple-tone interfering signals on a direct-sequence spread-spectrum communication system," IEEE Trans. Comm.un., vol. 30, pp. 436-446, Mar. 1982.

[14] M. B. Pursley, "Spread-spectrum multiple-access communications," in Multi-User Communication Systems, G. Longo, Ed. New York: Springer-Verlag, 1981, pp. 139-199.

[15] M. K. Simon, J. K. Omura, R. A. Scholtz, and B. K. Levitt, Spread Spectrum Communications, vol. II. Rockville, MD: Computer Science, 1985.

[16] R. Vijayan and H. V. Poor, "Nonlinear techniques for interference suppression in spread-spectrum systems," IEEE Trans. Commun., vol. 38, pp. 1060-1065, July 1990 .

[17] J. M. Wozencraft and I. M. Jacobs, Principles of Communication Engineering. New York: Wiley, 1965.

Post-print standardized by MSL Academic Endeavors, the imprint of the Michael Schwartz Library at Cleveland State University, 2014 